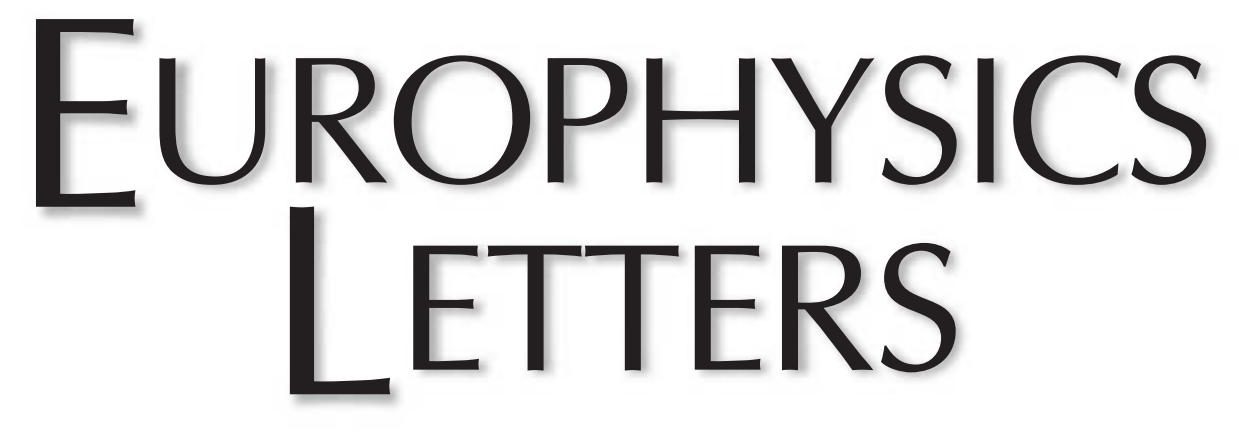

OFFPRINT

Vol. $65 \bullet$ Number $5 \bullet$ pp. $633-639$

Opening up fractal structures of three-dimensional flows via leaking

$* * *$

I. Tuval, J. Schneider, O. Piro and T. TéL

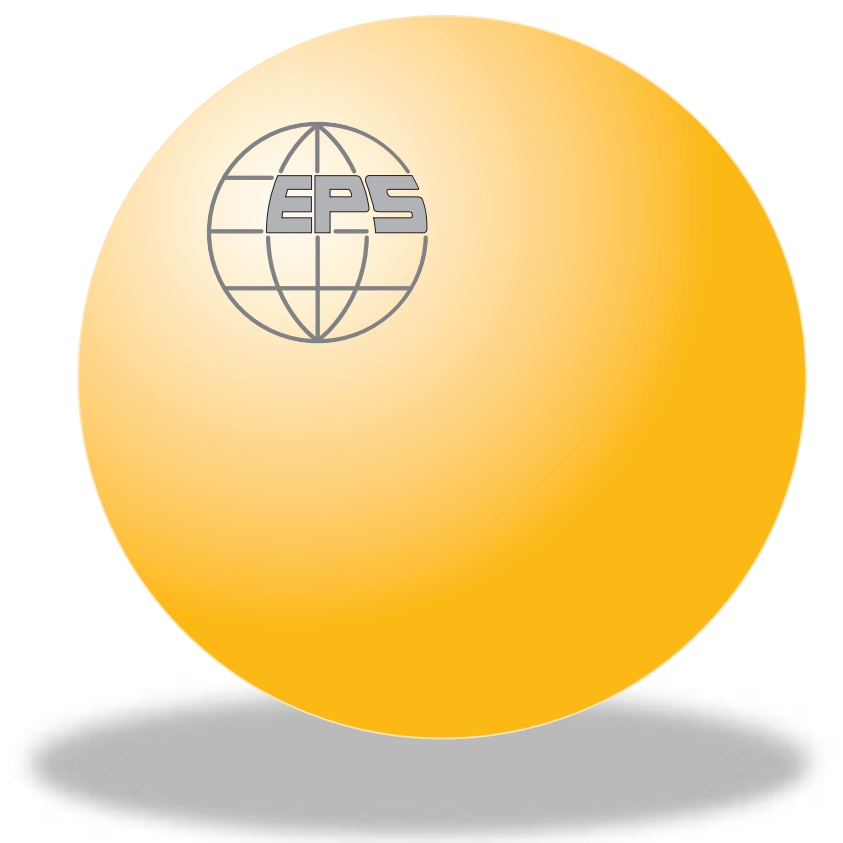

Published under the scientific responsibility of the

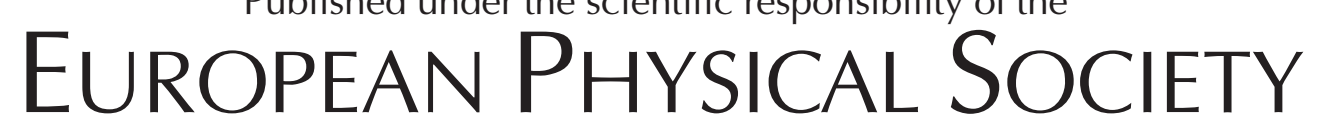

Incorporating

JOURNAL DE PHYSIQUE LETTRES • LETTERE AL NUOVO CIMENTO 


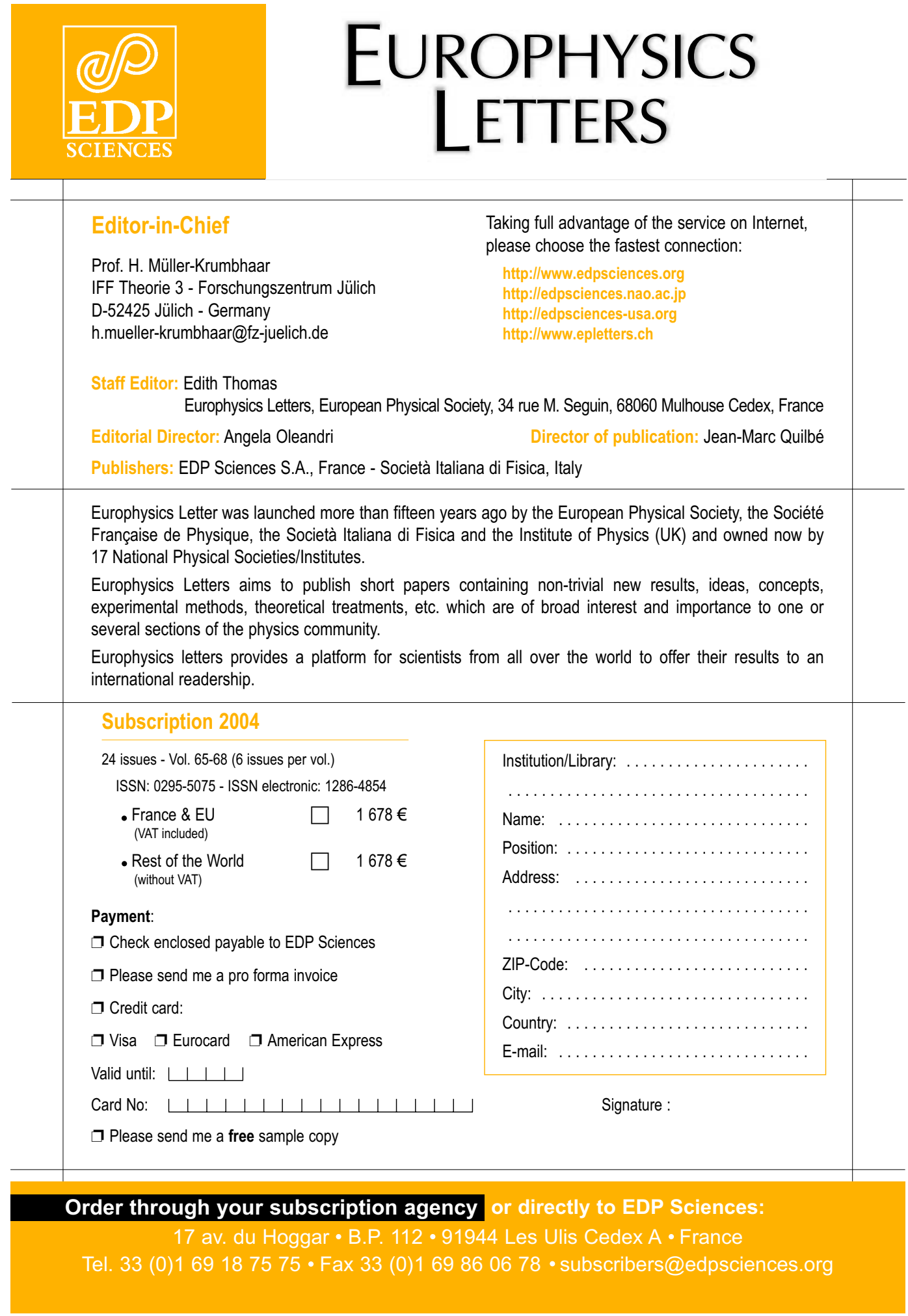


Europhys. Lett., 65 (5), pp. 633-639 (2004)

DOI: $10.1209 / \mathrm{epl} / \mathrm{i} 2003-10177-0$

\title{
Opening up fractal structures of three-dimensional flows via leaking
}

\author{
I. Tuval ${ }^{1}$, J. Schneider ${ }^{2}$, O. Piro ${ }^{1}$ and T. TÉL ${ }^{3}$ \\ 1 Institut Mediterrani d'Estudis Avançats, CSIC-UIB \\ E-07071 Palma de Mallorca, Spain \\ 2 Institut für Physik, Universität Potsdam \\ PF 601553, 14415 Potsdam, Germany \\ 3 Institute for Theoretical Physics, Eötvös University \\ Pf. 32, H-1538, Budapest, Hungary
}

(received 8 September 2003; accepted in final form 5 January 2004)

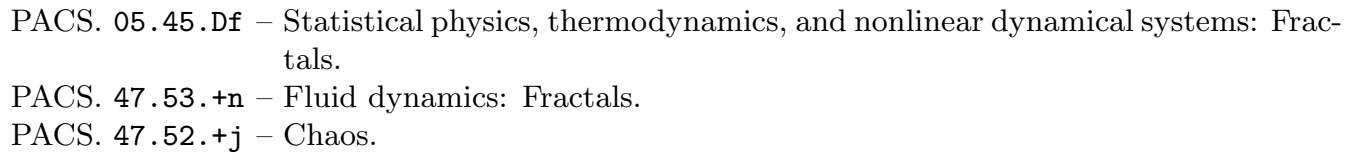

\begin{abstract}
We study the behavior of time-periodic three-dimensional incompressible flows modelled by three-dimensional volume-preserving maps in the presence of a leakage. The distribution of residence times, and the chaotic saddle together with its stable and unstable invariant manifolds are described and characterized. They shed light on typical filamentation of chaotic flows whose local stable and unstable manifolds are always of different character (plane or line). We point out that leaking is a useful method which sheds light on typical filamentation of chaotic flows. In particular, the topology depends on the number of local expanding directions, and is the same in the leaked system as in the closed flow.
\end{abstract}

Introduction. - The dynamics of passive impurities transported by incompressible fluid flows is conservative, the volume occupied by the particles remains constant under the evolution. There are situations, however, in which a peculiar type of dissipation comes to the scene. Consider, for example, the following problem: the air of a room in which a controlled flow pattern has been established needs to be cleaned. A purifier system installed at a specific location is to be chosen in order to maximize its efficiency. In a simplified view, we can think of the purifier as a volume of the space where the impurities are eliminated from the system without perturbing the flow. We are then facing a "leaking" dynamics in which trajectories evolve preserving the volume until they reach a specified region of the space where they disappear. Our problem is then deeply related to the investigation of the changes expected in the residence time of the particles in the allowed phase space - the room - as we modify the shape and location of the leak - the purifier design.

Manifestations of the same problem arise in very different and otherwise unrelated contexts. In mesoscopic physics, for example, the ballistic conductance of minute structures of both $2 \mathrm{D}$ and $3 \mathrm{D}$ character leads to conservative classical dynamics leaking through the feeding leads [1]. The problem is also relevant in other hydrodynamical contexts such as, e.g., the (c) EDP Sciences 
mixing properties of open flows [2]. In the realm of oceanographic research, the leaking could be related to the loss of planktonic material due to its predation by larger species. Chemical reactions occurring at certain regions of the flow might also be responsible for the deactivation/annihilation of the particles arriving in these regions, modelled by the so-called resetting mechanism [3].

Leaking dynamics has recently been studied in the Hamiltonian framework [4,5]. In particular, leaking two-dimensional area-preserving maps have been recently investigated thoroughly as a function of the shape, location and orientation of the leaking regions as well as the characteristics of the basic dynamical system [6]. Fluid-dynamically, these results apply to flows that are two-dimensional and possibly periodically time dependent. Although this case is important for some applications to geophysics, most of real-life situations lead to three-dimensional incompressible flows.

The purpose of this paper is to perform the first study of this kind for a conservative but non-Hamiltonian dynamical system, namely, volume-preserving three-dimensional maps describing the basic features of incompressible time-periodic flows. Apart from the applications, the study of leaked volume-preserving maps in three dimensions poses a few challenging questions to the theory of dynamical systems. For example, a key dynamical object to understand leaked systems as well as many other open dynamical systems such as those describing scattering processes, are the so-called chaotic saddles and their associated stable and unstable manifolds. The chaotic saddle represents the set of points that never escape through the leak either forward or backward in time. The stable (unstable) manifold is the union of points which converge towards the chaotic saddle in the forward (backward) dynamics, and can also be interpreted as the complement of the union of all the pre-images (images) of the leak.

In 2D Hamiltonian systems the preservation of volume implies that the two Lyapunov exponents must be equal in magnitude and opposite in sign. Consistently with this, the invariant manifolds of the leaked system present some sort of statistical time-reversal symmetry. For example, the fractal dimension of the stable and of the unstable manifolds are the same. New to 3D maps, however, is the fact that generically, the maps present either two positive and one negative expansion rates or viceversa. Consequently, a leaked $3 \mathrm{D}$ map is not a direct analog of a scattering problem since it is non-Hamiltonian, although the original system is volume preserving, but only for a particular choice of parameters - when one of the Lyapunov exponents is exactly zero - is it also time-reversal invariant. Here we investigate the impact of these features on the dimensionality and other statistical properties of the filamentation. Some of these questions turn out to have unexpected importance from the point of view of applications.

An interesting case, for example, is the analysis of magma flows from petrological data. Lavas often present a freezed form of the Lagrangian structures of these flows that can then be studied by image processing. Part of the processing may consist of band-pass filtering the grey-scale levels of the images which is an indirect way of introducing leaking. Some empirical results [7] show that the resulting fractal dimensions of the structures are strongly correlated with the mixing efficiency of the corresponding magma flows. In this case, however, leaking does not imply a physical escape out of the system, rather it means that regions with a certain property (e.g., grey-scale level) are filtered out. Considering the concentration as the filtering criterion, the same approach can be applied to the analysis of chemical reactions in flows [6].

As a paradigm of this type of three-dimensional systems, we use the family of $A B C$ and $A B C$-related maps [8]: $\boldsymbol{T}\left(x_{n}, y_{n}, z_{n}\right) \rightarrow\left(x_{n+1}, y_{n+1}, z_{n+1}\right)$, with

$$
\begin{aligned}
& x_{n+1}=x_{n}+A \sin \left(z_{n}\right)+C^{\prime} \cos \left(y_{n}\right), \\
& y_{n+1}=y_{n}+B \sin \left(x_{n+1}\right)+A^{\prime} \cos \left(z_{n}\right) \quad(\bmod 2 \pi), \\
& z_{n+1}=z_{n}+C \sin \left(y_{n+1}\right)+B^{\prime} \cos \left(x_{n+1}\right) .
\end{aligned}
$$



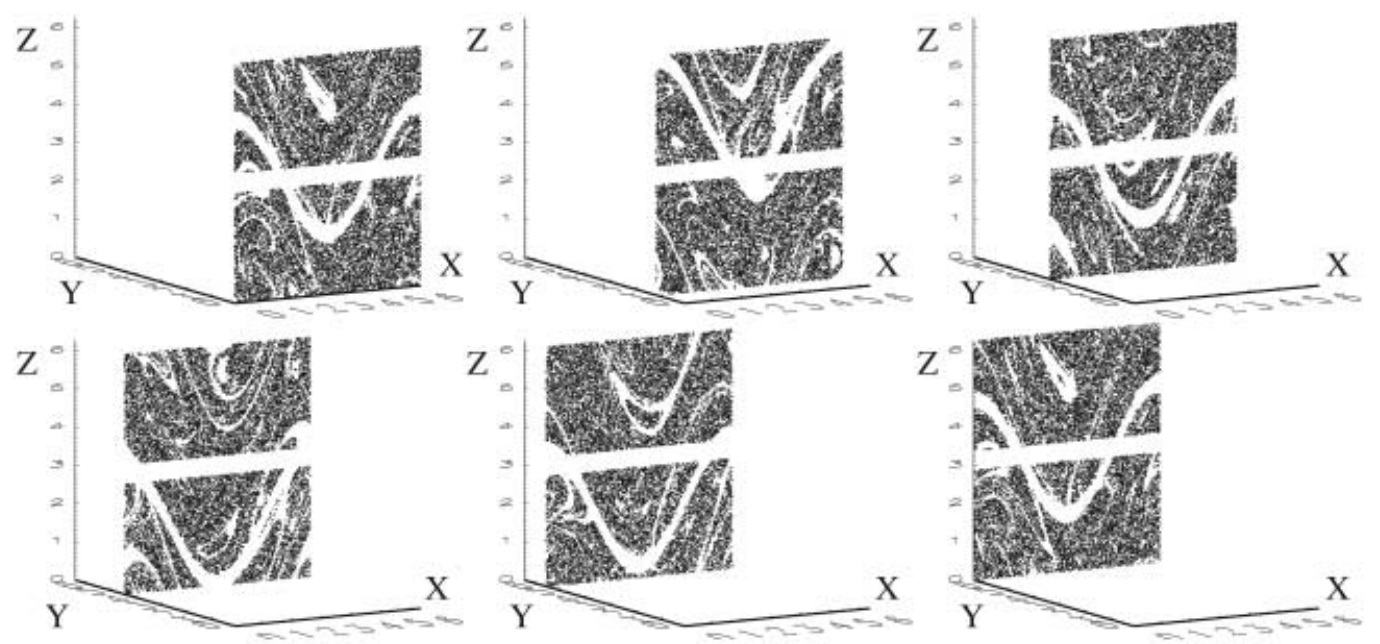

Fig. $1-X Y$ slices of the chaotic saddle for the leaked $A B C$ map with $A=B=C=1.6$. The leak is centered at $z=\pi$ with a width of $\epsilon=0.5$. The number of initially distributed particles is $N_{0}=5000000$. The width of slices is $0.08 \pi$.

Although the $A B C$ map is the solution of the Navier-Stokes equation in the presence of a rather artificial space-time periodic forcing [9], it has been shown to capture the essential features of more realistic three-dimensional time-periodic flows [10-12] whose geometries are relevant to geophysical and environmental applications. This family of maps display a range of dynamical behavior that goes from nearly integrable to strongly chaotic ones that in some sense can be considered the equivalents in three dimensions of those found in even-dimensional conservative Hamiltonian dynamics.

In the following, we will focus on the highly chaotic case, where no large-scale sticky integrable regions are found numerically, and we will investigate the effect of introducing leaking regions. As an example, we define a rectangular box lying parallel to the $(x, y)$-plane of width of $\epsilon=0.5$, centered at $z=\pi$, cutting through the whole system in $z$, as the leak. Particles which enter the leak during the simulations escape the system. Remaining particles then trace out fractal structures, which have a dimension less than 3 , the dimension of the whole accessible space.

Starting with a large number $N_{0}$ of particles initially distributed in the full phase space uniformly, all the nonescaping particles are followed over $n$ number of iterations. For large enough $N_{0}$ and $n$, the initial coordinates of the nonescaping particles should trace out the stable manifold, their final positions after $n$ steps the unstable manifold, and the midpoints at time $n / 2$ the chaotic saddle itself [13].

For iterating $n=17$ times, we obtain the chaotic saddle as shown in fig. 1 . In order to have a better view on the three-dimensional structure, the chaotic saddle is represented in slices of the system centered at different $y$. It can be seen in the pictures that the sine structures appear both in the $x$ - and in the $z$-direction.

As a tool of visualizing dynamical properties of the particles in the leaked $A B C$ map, we calculated their residence times. A $2 \mathrm{D}$ slice of this function is depicted in fig. 2a: regions with large lifetimes (particles need long times to reach the leak) are denoting regions close to the stable manifold, thus the structures traced out by the long-living particles practically coincide 

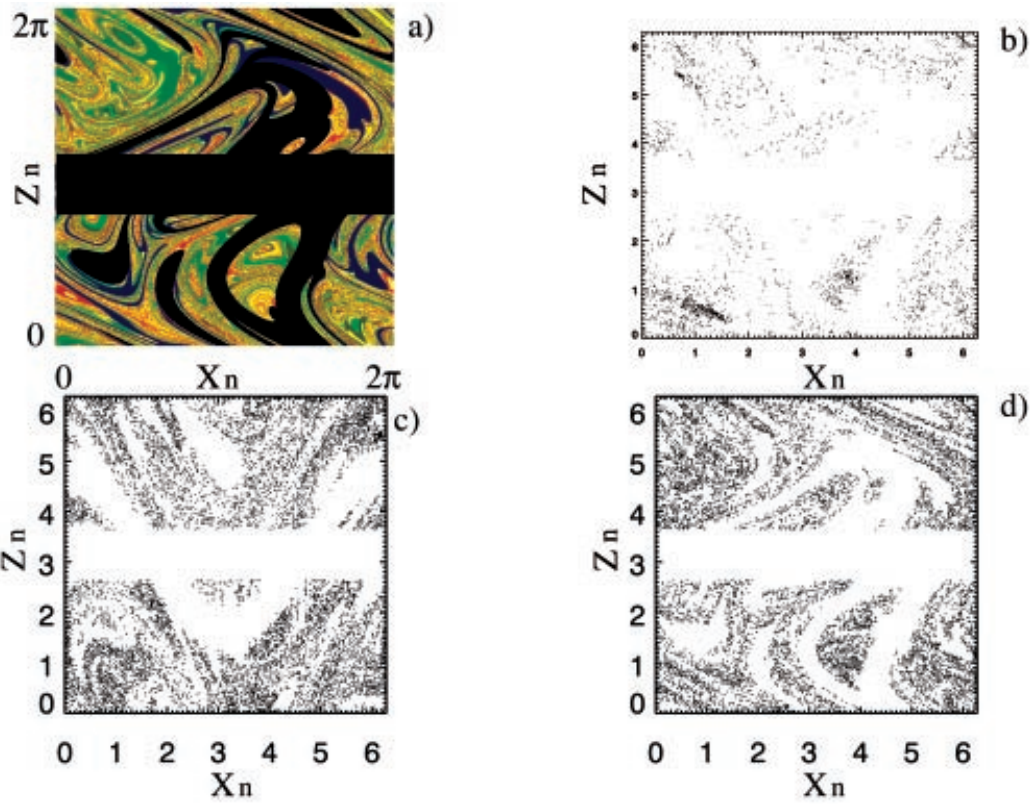

Fig. 2 - (a) $X Z$ slice of the residence time function for the same parameters as in fig. 1. The leak is centered at the same $z$-value but with a width of $\epsilon=1.0$. Dark colors mean small residence times, while light colors represent higher values. (b) $X Z$ slice of the chaotic saddle refined with the PIMtriple method. (c) and (d) the associated unstable and stable manifolds, respectively. The average mean residence time $\tau$ of the associated chaotic transient is used to obtain the fractal dimensions, and its value is $1 / \tau=0.192$. All the slices are for $y=0$.

with the ones of the stable manifold. The residence times depend sensitively on the size and on the position of the leak [6]. For a fixed size of the leak, such as the purifier, calculations of the residence or escape times will give the optimal location for an efficient particle exchange.

Figure $2 \mathrm{~b}$ depicts the chaotic saddle, refined with the PIM-triple method [14]. The method is based on the so-called PIM (Proper Interior Maximum) triples: three points ( $a, c, b)$ in a straight line segment such that the interior point $c$ has the maximum residence time. Once a PIM-triple is found, it is used to approach the stable manifold of the chaotic saddle, looking for refined segments in $(a, b)$ that contain closer PIM-triples. This constitute an iterative scheme to approach the stable manifold. Initial conditions on the stable manifold in the $X Z$ plane at $y=0$ are then evolved $n / 2$ times to obtain the chaotic saddle. A slice (with width $0.01 \cdot \pi$ ) of this three-dimensional saddle is plotted in the figure. Slices of its unstable and stable manifolds are plotted as well (figs. 2c, d, respectively). If the chaotic saddle has a large dimension, this implies that the emptying process is slow, that is, the location or size of the purifier is not optimal. The visualization of parts of its otherwise space-filling three-dimensional unstable manifold is an important step to obtain the stretching filamentation [15], i.e. the routes of efficient transport, in a system. Furthermore, visualizing different subsets of the unstable manifold by defining different leaks (different shapes, positions or sizes) corresponds to mimicking different concentration layers, traced out, e.g., by chemical or biological processes or by magma mixing processes.

We have computed the fractal dimensions of the unstable and stable manifolds of the chaotic saddle of the leaked map. We have used both the correlation dimension and the 
generalization of the Kaplan-Yorke formula for the information dimension. The generalization of the Kaplan-Yorke formula for $N$-dimensional maps [16] yields the information dimensions of the unstable, stable manifolds and of the saddle itself as

$$
\begin{aligned}
D_{\mathrm{u}} & =U+I+\left[\left[\left(\sum \lambda_{j}^{+}\right)-\frac{1}{\tau}\right]-\left(\left|\lambda_{1}^{-}\right|+\cdots+\left|\lambda_{I}^{-}\right|\right)\right] /\left|\lambda_{I+1}^{-}\right|, \\
D_{\mathrm{s}} & =S+J+\left[\left[\left(\sum \lambda_{j}^{+}\right)-\frac{1}{\tau}\right]-\left(\lambda_{1}^{+}+\cdots+\lambda_{J}^{+}\right)\right] / \lambda_{J+1}^{+}, \\
D_{\text {saddle }} & =D_{\mathrm{u}}+D_{\mathrm{s}}-N .
\end{aligned}
$$

Here $\lambda_{i}^{+}$and $\lambda_{j}^{-}$are the positive and negative Lyapunov exponents, $\tau$ denotes the average residence time around the saddle, $U$ and $S$ are the number of positive and negative Lyapunov exponents, $N=U+S$, and $I$ and $J$ are such that the following relations are fulfilled:

$$
\begin{aligned}
\left|\lambda_{1}^{-}\right|+\cdots+\left|\lambda_{I+1}^{-}\right| & \geq\left[\left(\sum \lambda_{j}^{+}\right)-\frac{1}{\tau}\right] \geq\left|\lambda_{1}^{-}\right|+\cdots+\left|\lambda_{I}^{-}\right|, \\
\lambda_{1}^{+}+\cdots+\lambda_{J+1}^{+} & \geq\left[\left(\sum \lambda_{j}^{+}\right)-\frac{1}{\tau}\right] \geq \lambda_{1}^{+}+\cdots+\lambda_{J}^{+} .
\end{aligned}
$$

All the values listed below correspond to the mean values of the measured correlation dimension and the information dimension computed with eq. (2). We investigated the case $A=A^{\prime}=B=B^{\prime}=C=C^{\prime}=1.6$. Analogously to the $2 \mathrm{D}$ case, the stable and unstable manifolds have then the same dimension. We obtained

$$
\begin{aligned}
& \lambda_{1}=+0.775, \quad \lambda_{2}=+0.001, \quad \lambda_{3}=-0.776, \\
& D_{\mathrm{u}}=2.752, \quad D_{\mathrm{s}}=2.751, \quad D_{\text {saddle }}=2.50 \text {. }
\end{aligned}
$$

In the more interesting case when the Lyapunov exponents of the map are all nonzero, new characteristics of the manifolds appear in comparison to the $2 \mathrm{D}$ case. Now, the manifold related to the two exponents of equal sign (i.e. the unstable manifold if the system shows 2 positive Lyapunov's, and the stable one in the case of two negative Lyapunov's) is locally a surface, while the other one is a line. Due to the foldings generated by the chaotic dynamics, their global form is a curtain-like and a filamental fractal, respectively. The former possesses a larger dimension than the other one.

This can clearly be seen in a different volume-preserving map, a 3D toral automorphism:

$$
\left(\begin{array}{l}
x_{n+1} \\
y_{n+1} \\
z_{n+1}
\end{array}\right)=\left(\begin{array}{lll}
1 & 1 & 2 \\
1 & 3 & 5 \\
2 & 5 & 9
\end{array}\right) \cdot\left(\begin{array}{l}
x_{n} \\
y_{n} \\
z_{n}
\end{array}\right) \quad(\bmod 1)
$$

This map is a constant matrix hyperbolic map whose Lyapunov exponents can be obtained analytically as the logarithm of the matrix eigenvalues. The fractal dimension of the invariant manifolds is consistent with the Lyapunov values. Slices of the stable and unstable manifolds are depicted in fig. 3a, b: $\lambda_{1}=+2.5, \lambda_{2}=-0.53, \lambda_{3}=-1.97, D_{\mathrm{u}}=2.315, D_{\mathrm{s}}=2.857$, $D_{\text {saddle }}=2.172$.

For the nonlinear $A B C$ map, these manifolds are no longer translation invariant. In the particular case of the $A B C$ parameters as $A=3.6, A^{\prime}=1.6, B=4.6, B^{\prime}=0.06, C=5.06$, 


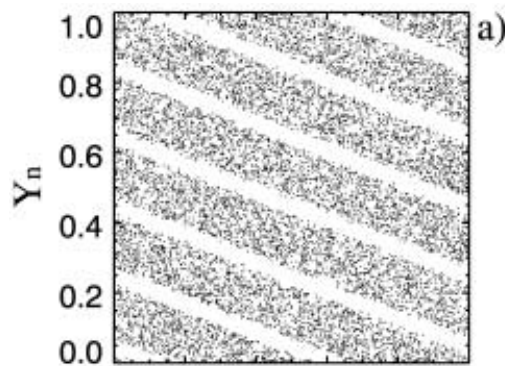

$\begin{array}{llllll}0.0 & 0.2 & 0.4 & 0.6 & 0.8 & 1.0\end{array}$ $\mathrm{X}_{\mathrm{n}}$

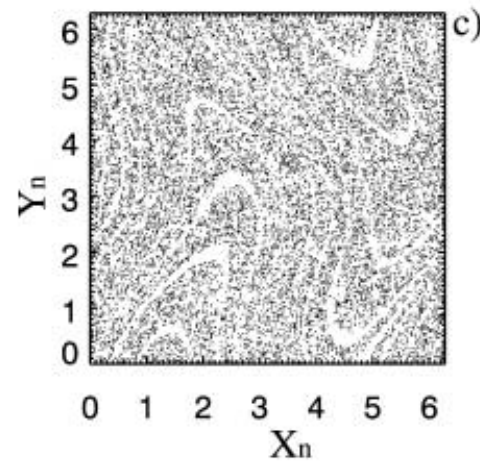

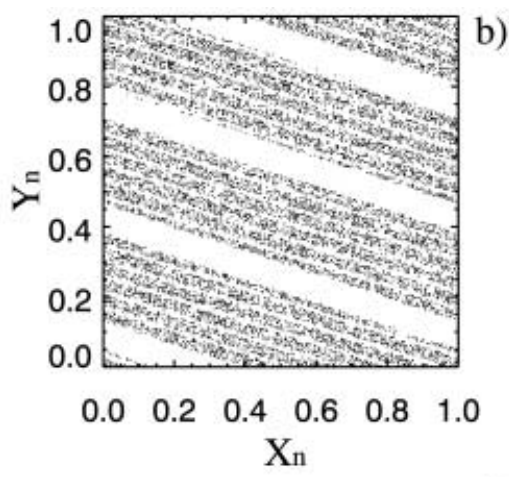

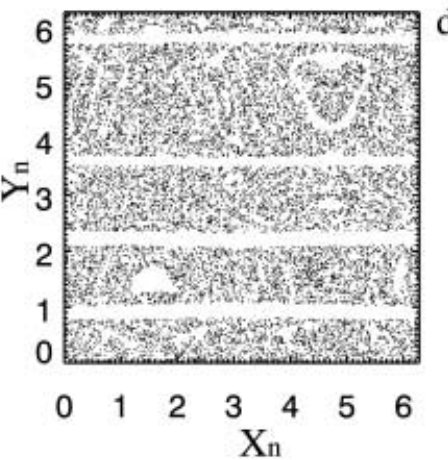

Fig. $3-X Y$ slices (for a value $z=0.8$ and $z=0.8 \cdot \pi$, above the leak) of the stable and unstable manifolds for the 3D toral automorphism (a, b) and for the asymmetric $A B C$ map (c, d). The leak is a rectangular box parallel to the $X Y$ plane and centered at $z=0.3(z=0.3 \cdot \pi)$ with a width of $\epsilon=0.25(\epsilon=0.25 \cdot \pi)$. The width of the slices is $0.01(0.01 \cdot \pi)$. The mean residence time values are $1 / \tau=0.357$ and $1 / \tau=0.227$, respectively.

$C^{\prime}=0.6$, the map has two negative and one positive exponent. Consequently, the dimension of the stable manifold is larger than that of the unstable manifold:

$$
\begin{array}{cc}
\lambda_{1}=+2.53, \quad \lambda_{2}=-0.9, \quad \lambda_{3}=-1.63 \\
D_{\mathrm{u}}=2.864, \quad D_{\mathrm{s}}=2.91, \quad D_{\text {saddle }}=2.774 .
\end{array}
$$

The topological difference of both manifolds is visible in these approximately two-dimensional slices (fig. 3c, d). The stable one appears to be filamental in this cut, while the unstable one is a cloud of points without any systematic filamentation consistently with the lower fractal dimension of the latter manifold.

In conclusion, the proposed application of the method of leaking is shown to provide insight into the phase space structures of chaotic 3D incompressible flows. In the example of the $A B C$ map, which represents a general class of 3-dimensional systems, it is shown to be a fast and suitable way in quantifying physical and geophysical systems.

The performed example of measuring the fractal dimension in the highly chaotic $A B C$ map with 3 nonzero Lyapunov exponents is in agreement with the theory: the manifold related to the Lyapunov exponents with equal sign has the larger dimension.

Fractal structures have recently been shown [7] to be quantifiers of mixing efficiency in magma processes. Until now, similar structures have numerically been reproduced only by 
following a dye droplet in closed 3D flows, which, however, do not possess a fractal dimension other than 3. Our method shows that by leaking the flow, filaments of a clean fractal dimension appear which are even closer to empirical observations.

OP and IT acknowledge the Spanish Ministerio de Ciencia y Tecnologia, Proyecto CONOCE, contract BFM2000-1108 and Proyecto IMAGEN, contract REN2001-0802-C02-01. JS acknowledges financial support from the International Max Planck Research School for Biomimetic Systems (IMPRS). TT is supported by OTKA T032423.

\section{REFERENCES}

[1] Sim H. S. and Schomerus H., Phys. Rev. Lett., 89 (2002) 066801.

[2] Jung C., TÉL T. and Ziemniak E., Chaos, 3 (1993) 555.

[3] Pierrehumbert R. T., Chaos, Solitons Fractals, 4 (1994) 1091.

[4] Motter A. E. and Letelier P. S., Phys. Lett. A, 285 (2001) 127.

[5] Schneider J. and TÉL T., Ocean Dynam., 53 (2003) 64.

[6] Schneider J., Tél T. and Neufeld Z., Phys. Rev. E, 66 (2002) 066218.

[7] Perugini D., Poli G. and Gatta G. D., Lithos, 65 (2002) 313.

[8] Feingold M., Kadanoff L. P. and Piro O., J. Stat. Phys., 50 (1988) 529.

[9] Galluccio S. and Vulpiani A., Physica A, 212 (1994) 75.

[10] Cartwright J. H. E., Feingold M. and Piro O., Physica D, 76 (1994) 22.

[11] Cartwright J. H. E., Feingold M. and Piro O., Phys. Rev. Lett., 75 (1995) 3669.

[12] Cartwright J. H. E., Feingold M. and Piro O., J. Fluid Mech., 316 (1996) 259.

[13] Lai Y.-C., TÉl T. and Grebogi C., Phys. Rev. E, 48 (1993) 709.

[14] Nusse H. E. and Yorke J. A., Physica D, 36 (1989) 137.

[15] Ottino J. M., The Kinematics of Mixing: Stretching, Chaos, and Transport (Cambridge University Press) 1989.

[16] Hunt B. R., Ott E. and Yorke J. A., Phys. Rev. E, 54 (1996) 4819. 